\title{
A escrita cursiva romana
}

\author{
PEDRO PAULO A. FUNARI \\ Departamento de História \\ Instituto de Filosofia e Ciências Humanas \\ Universidade Estadual de Campinas
}

\begin{abstract}
RESUMO: Este artigo apresenta ao leitor as principais questões referentes à escrita cursiva romana. Trata da chamada Escrita Cursiva Romana Antiga, das capitais cursivas e das cursivas especializadas usadas por escribas profissionais. Também menciona a inevitável ligação entre paleografia e interpretação semântica e ressalta a materialidade da escrita.
\end{abstract}

PALAVRAS-CHAVE: Escrita romana; cursiva romana antiga; capitais; paleografia.

Jean Mallon pode ser considerado, a justo título, o fundador da moderna paleografia romana, tendo sido sua obra considerada, por Tjäder, eine Revolution in der Geschichte der lateinischen Schriftsforschung ("uma revolução na História da pesquisa da escrita latina") (Bowman \& Thomas, 1983, p. 53-55). Mallon (Mallon, 1952, p. 105), contudo, preferiu criar uma nova expressão para designar a escrita quotidiana dos romanos, écriture commune classique, caracterizada, por oposição à escrita capital, como a maneira de escrever do dia a dia, não necessariamente "rápida", como a palavra "cursiva" deixa transparecer (cursus = "movimento rápido"). Embora suas interpretações revolucionárias tenham sido, em grande medida, incorporadas pela moderna paleografia romana, o termo "escrita comum" acabou preterido, prevalecendo a expressão tradicional "escrita cursiva" (Kursivschrift). Esta pode ser definida como uma "escrita de mão" (hand writing), por oposição à escrita monumental, feita com instrumentos mais ou menos duros, como o estilete (graphio) ou o calamus, com ou sem tinta.

Distiguem-se a escrita romana cursiva antiga, usada até meados do terceiro século d.C., e a escrita cursiva romana nova, introduzida por essa época (fig. 1-2). Aceita-se, hoje, que a forma das letras cursivas antigas derivam das capitais e que a chave para a compreensão dessas letras está nos traços que as compõem e na inclinação do instrumento que escreve. A forma das letras podia alterar-se pela posição, inicial ou final, por efeito estético, por contignatio, ou pelo uso de abreviaturas ou siglas. Tem chamado atenção aos estudiosos da escrita romana a sua uniformidade em um espaço geográfico tão grande como era o Império Romano. Letras de Pompéia, Roma, Vindolanda, Aquae Sulis (Bath, na Inglaterra) (fig. 36) na fronteira noroeste, apresentam mais semelhanças do que diferenças, o que está a indicar a existência de mecanismos de homogeneização sem paralelos no período pré-moderno (fig. 7-15). Nesta breve apresentação da escrita cursiva romana, tratarei em primeiro lugar, a partir da minha experiência com a publicação de inscrições cursivas inéditas e do estudo e 
tradução de epígrafes publicadas, das características substanciais das cursivas antigas. A composição das letras, com traços feitos em ordem fixa, constitui elemento central de estudo da escrita romana. Note-se como a letra B exige que o traço da esquerda seja delineado antes do traço superior. Ou ainda, como a letra O compõe-se de dois traços.

A compreensão do movimento ou ductus da mão é essencial pois, na prática, muitos "alongamentos", à esquerda ou direita, explicam-se pelo ductus, como pode notar-se nas cursivas pintadas e nos grafites anfóricos (fig. 7-8). As inscrições em ânforas oleárias Dressel 20 foram feitas na Bética, província na Espanha Meridional e, nem por isso, suas letras distanciam-se daquelas usadas em outras partes. É verdade que podia haver cursivas "técnicas", como é o caso da notação de números, neste caso o peso do vaso ou do azeite, em libras romanas, que se utilizavam de um estilo conhecido, desde o século passado, como notae hispanicae ("letras espanholas"), cuja leitura, no entanto, devia ser ainda mais fácil, por parte dos oficiais controladores (fig. 9-13). Capitais cursivas, como em uma ânfora Dressel 20, que estão a identificar a societas Aemiliorum et Cassiorum, que publiquei há algum tempo, são facilmente legíveis, ainda hoje (fig. 11). Outras, como as cursivas minúsculas na mesma peça, destinadas a funcionários, com abreviaturas e siglas, não se destinavam ao público geral e eram mais técnicas (aaaa por arca, por exemplo) (fig. 12).

Outra característica importante da cursiva antiga é a separação das palavras, algo que seria abandonado na cursiva nova, na qual predomina a scriptio continua. Os interstícios facilitavam muito a leitura, como se pode observar por uma carta proveniente de Vindolanda (fig. 14). Este caráter didático da escrita cursiva antiga fica ainda mais claro nos grafites pompeianos, como CIL IV, 1331 (martialis cunuligus), 4764 (perari fur es), 5370 (fortuna), 4755 (cresces architectus), 4756 ( $q$ habiat clymene(n)), 5372 (sum tuaa ae(ris) a(ssibus) II) (fig. 15).

Heikki Solin (Solin, 1970, p. 9), estudioso dos grafites antigos, afirmava, como ponto de partida da compreensão das inscrições comuns romanas, que se devia partir da filologia para explicar a paleografia:

Voglio dire che quando ci si propone di spiegare un qualsiasi graffito, non si può cominciare col dare a ciascun segno un valore grafico e vedere poi che contenuto ne risulti. Noi dobbiamo invece cercare dapprima di spiegarci, con criteri filologi, cosa lo scrivente abbia voluto dire, e solo poi domandarci quale sia il valore del risultado paleografico raggiunto dallo scrivente e cioè quale sia il suo livello di abilità grafica. Solo per ultimo possiamo analizzare che cosa il graffito in questione possa rappresentare per la storia della scritura. In: altre parole, voglio sottolineare che, nello studio dei graffiti, l'interpretazione 'filologica' va portata prima di un interpretazione 'paleografica'. Come si può credere che uno scrivente semianalfabeta esguisse i suoi caratteri proprio secondo gli schemi del modello corsivo o, nel caso capitale, delle singole lettere? Confondere tra loro due lettere abbastanza simili, como B e D, A ed R ecc, ci sembra molto comprensibile quando a farlo siano state persone che non dominavano completamente il mezzo grafico. In: simili casi la lettura va ragionevelmente condotta con criteri puramente filologici.

"Quero dizer que, quando nos propomos a explicar um grafite qualquer, não se pode começar com dar a cada signo um valor gráfico e ver, 
depois, que conteúdo resulte. Devemos, ao contrário, buscar, antes, explicar, com critérios filológicos, o que o escritor tenha querido dizer e, só depois, perguntar-nos qual tenha sido o valor do resultado paleográfico atingido pelo escritor, ou seja, qual era seu nível de habilidade gráfica. Só ao final podemos analisar o que o grafite em questão possa representar para a história da escrita. Em outros termos, quero ressaltar que, no estudo dos grafites, a interpretação 'filológica' tem precedência sobre a interpretação 'paleográfica'. Como se pode acreditar que um escritor semi-analfabeto escrevesse os caracteres segundo os esquemas do modelo cursivo ou, no caso das capitais, das letras isoladas? Confundir letras bastante semelhantes, como B e D, A e R etc., parece-nos muito compreensível, quando quem o faz são pessoas que não dominam completamente o meio gráfico. Em tais casos, a leitura deve ser conduzida a partir de critérios puramente filológicos".

No entanto, a subjetividade implícita na proposta fillológica de Solin dificulta sua adoção integral, pois podemos estar a transpor nossas idéias para o mundo antigo. Os exemplos de grafites aqui apresentados, não por acaso, apresentam diferenças notáveis de leitura, como CIL IV, 1331, lido por Mommsen martialis gunul gus e por Mau e Garrucci martialis cunuligus, CIL IV, 4764, na primeira linha, teríamos peram, perarl ou perari. A leitura, na verdade, exige seja um exame paleográfico formal, seja propostas de interpretação filológica. Assim, a respeito de CIL IV, 4756, a análise do estudo dos traços permite, segundo parece, sugerir a leitura habiat por habeat, como indicam outros exemplos (CIL IV, 538, 2083; cf. Väänänen, 1937, p. 62, sobre as desinências verbais -io, ias etc por -eo, -eas etc.). Pode concluir-se, assim, que o estudo da cursiva romana não pode prescindir de um estudo, a uma só vez, filológico, já que se trata de mensagens verbais, paleográfico, pois era uma forma de escrita quotidiana, e arqueológico, pois sua forma material exige técnicas de exegese física (local de achado, material de base, instrumento usado para a escrita, técnica de recuperação das imagens e de diferenciação de letras e elementos físicos naturais da superfície, entre outros) ${ }^{1}$. Considerando-se o imenso manancial de textos em cursivo já conhecido e, constantemente, dado à luz da ciência, podem entrever-se perspectivas fecundas para o campo do estudo das cursivas romanas.

\section{Nota}

1 - Ao final, R. Treuil, W. Burckert, H. Sarian, N.L. Guarinello e P.P.A. Funari convergiram quanto à necessidade de que arqueólogos, historiadores, filólogos e outros especialistas sobre a Antigüidade Clássica, mantenham-se em contato pois, apenas dessa forma, os resultados serão menos parciais e lacunosos, em particular no que se refere ao uso da escrita.

\section{Referências bibliográficas}

BOWMAN, A. K. \& THOMAS, J. D. 1983 Vindolanda: the Latin writing-tablets. Londres: Classical Association, 1983. 
BOWMAN, A.K. The Roman writing tablets from Vindolanda. Londres: British Museum, 1983.

FUNARI, P. P. A. Dressel 20 amphora inscriptions found at Vindolanda: the reading of the unpublished evidence. In: V.A. Maxfield \& M.J. Dobson (eds), Roman Frontier Studies 1989. Exeter: Exeter University Press, 1991. p. 65-72.

Some Roman inscriptions from Britain: amphora evidences. Revista do Museu de Arqueologia e Etnologia da Universidade de São Paulo. São Paulo, 3, p. 121-135, 1993.

MALLON, J. Paléographie Romaine. Madri: Consejo Superior de Investigaciones Científicas, 1952.

SOLIN, H. L'Interpretazione delle iscrizioni parietali, note e discussioni. Faenza: Edizioni Academiche, 1970.

TOMLIN, R. S. O. The curse tablets. In: Cunliffe, B. (Ed.). The Temple of Sulis Minerva at Bath, volume 2, The finds from the sacred spring. Oxford: Oxford Committee for Archaeology, 1988. p. 59-278.

VÄÄNÄNEN, V. Le Latin vulgaire des inscriptions pompéiennes. Helsinki: Academiae Scientiarum, 1937.

FUNARI, P. P. A. Roman cursive writing. Classica, São Paulo, 11/12, 213-230, 1998/1999.

ABSTRACT: The paper introduces the reader to the main issues regarding the Roman cursive writing. It deals with the so-called Old Roman Cursive (ORC), the cursive capitals and the specialized cursives used by professional scribes. It also mentions the unavoidable link between paleography and semantic interpretation and stresses the materiality of writing.

KEY-WORDS: Roman writing; old Roman cursive; capitals; paleography.

\section{Agradecimentos}

Agradeço aos seguintes colegas: A.K. Bowman, E. Rodríguez-Almeida, H. Solin, J.D. Thomas, R.S.O. Tomlin. A responsabilidade pelas idéias restringese ao autor. 


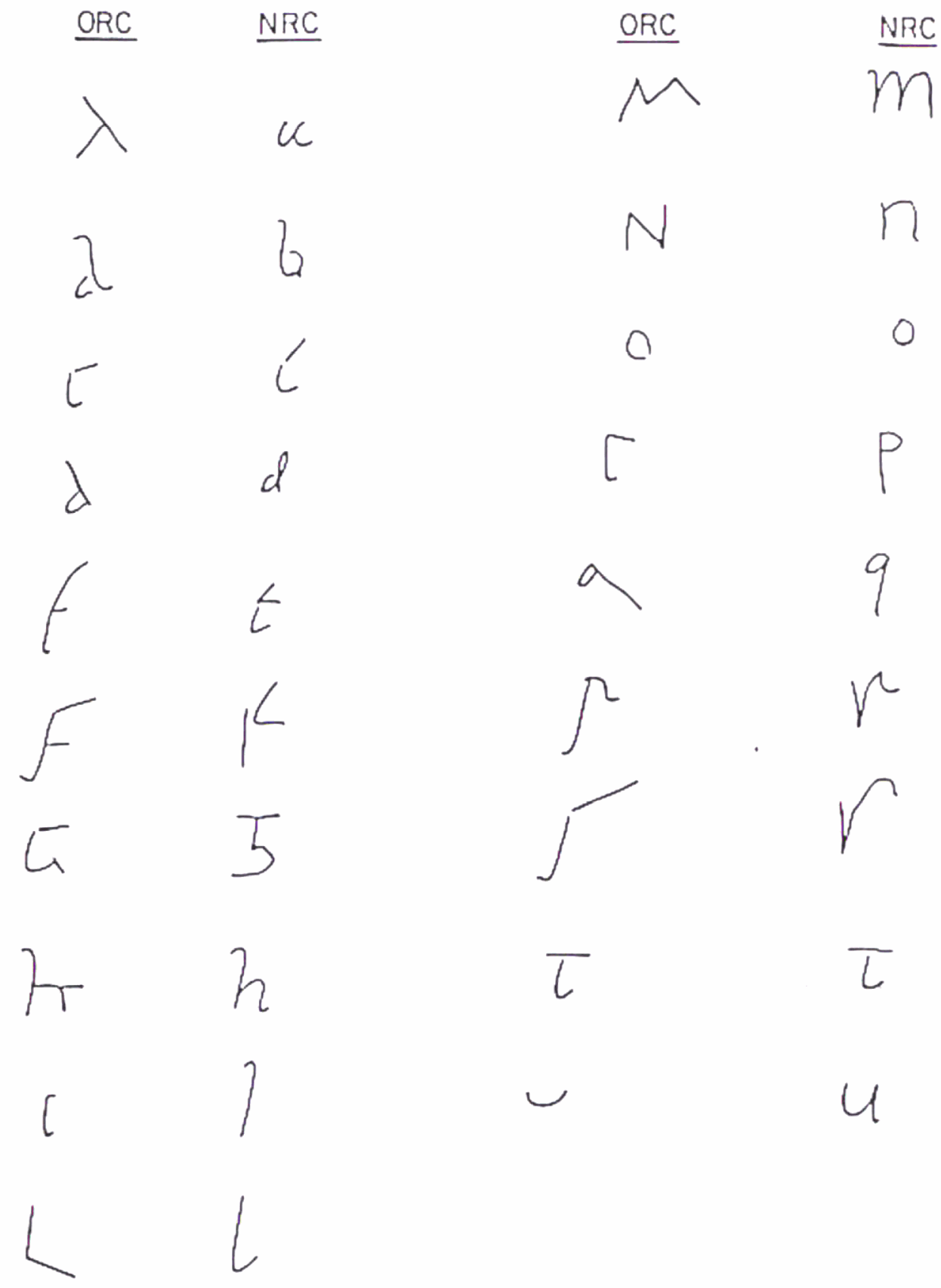

Fig. 1 - Tabletes de Vindolanda (Bowman \& Thomas, 1983, fig.10). 

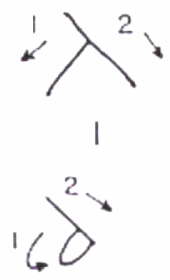

7

1

13
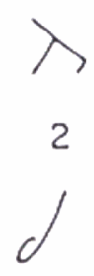

8

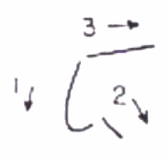

14
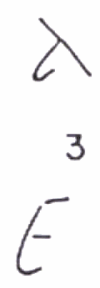

9
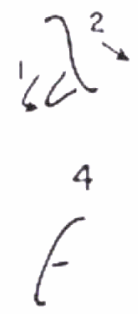

10

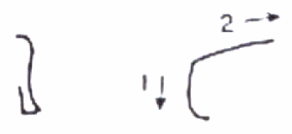

6

II

$177^{3}=$

15

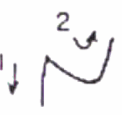

21

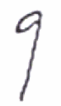

26

27

$\downarrow$

33
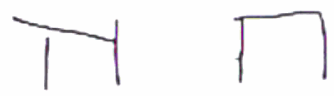

[

16
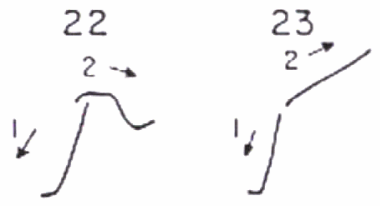

28

29

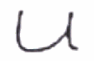

34

Fig. 2 - Tabletes de Vindolanda (Bowman \& Thomas, 1983, fig.11). 


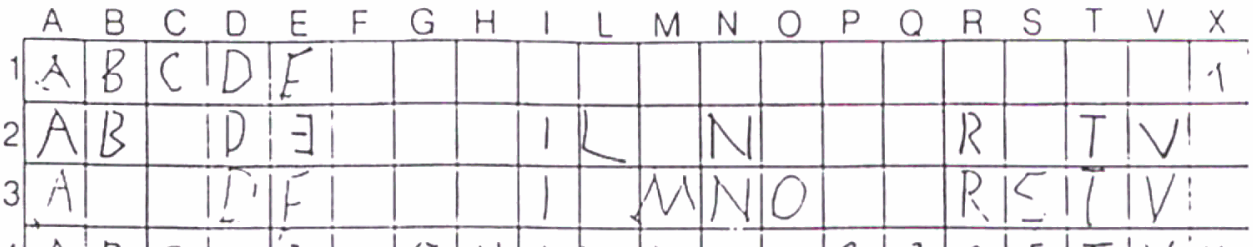

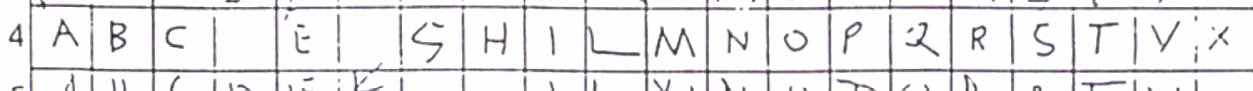
5 A BLLWEIE 6 A IDIEI S ILMNI RRISITV

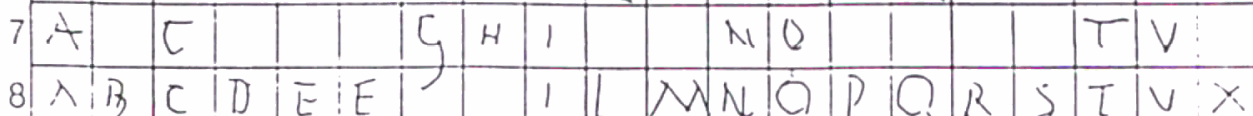

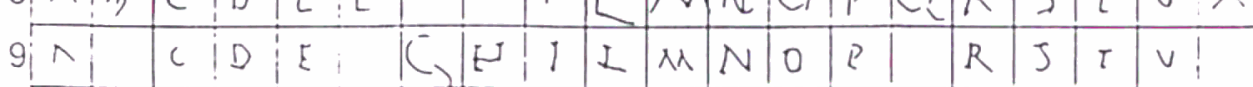

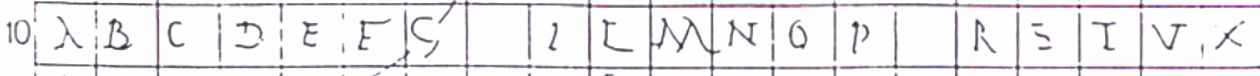

\begin{tabular}{|c|c|c|c|c|c|c|c|c|c|c|c|c|c|c|c|c|c|}
\hline $11(1) \mid$ & & $c$ & & & IE & & $\bar{I}$ & & & & & & $Q$ & $R$ & $j$ & $E$ & $v \mid$ \\
\hline $11(11)$ & $\therefore 18$ & & $D$ & 15 & & & $1 !$ & & & & & & & $R$ & & $I$ & \\
\hline IIII & & ( & i & & & & I & & $M$ & & & & $Q$ & & & & $\because$ \\
\hline $2(1)$ & & & & $\mid c$ & & & 1 & 2 & $M$ & $N$ & $a$ & & Q & $R$ & S & $\Gamma$ & $E$ \\
\hline 2 & & & B. & | & & & & & $\mu$ & & & & & $R$ & s & & \\
\hline (11) & & & & 8 & & & 1 & 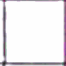 & $M$ & $N$ & & & & & I & $T$ & \\
\hline 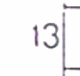 & & & $D$ & S & & & 1 & 2 & $\wedge$ & $N$ & 0 & & & $R$ & 5 & $T$ & $\checkmark$ \\
\hline 14 & $\stackrel{\wedge}{\circ}$ & & $6: 0$ & $z$ & & & 1 & $<$ & $\Lambda x$ & 4 & (1) & & & a & $s$ & $T$ & $\checkmark T$ \\
\hline 15 & & & 0 & 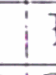 & & & 1 & $<$ & $x^{x}$ & $N$ & 0 & & $P$ & $R$ & 3 & $T$ & $V$ \\
\hline 16 & & & E & 10 & & & 1 & 1 & $\lambda$ & 1 & C? & & 0 & $R$ & 5 & $T$ & 1,1 \\
\hline 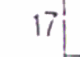 & & & $=11$ & 18 & & $1 \mathrm{i}$ & $1 /$ & $L$ & $i v$ & $N$ & 0 & & & 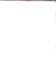 & S & $T$ & Y \\
\hline 18 & & & 13 & II & & & 11 & & $x$ & H & $C_{1}$ & & & h & & & $|v|$ \\
\hline 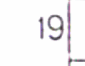 & $A$ & & $! ?$ & 1 & & & $1:$ & & M & 11 & & & & $R$ & S & $I$ & $\because 1$ \\
\hline $\mathrm{CO} \mathrm{O}_{1}$ & & & $i$ & 18 & & & 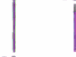 & & X & & & & 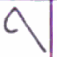 & & & $T$ & $\backslash 1$ \\
\hline 2 & & & & 12 & & & 1 & & & & & $P$ & & & $S$ & $T$ & $|v|$ \\
\hline
\end{tabular}

Fig. 3 - Tabulação de formas de letras (Tomlim, 1988, p. 91). 


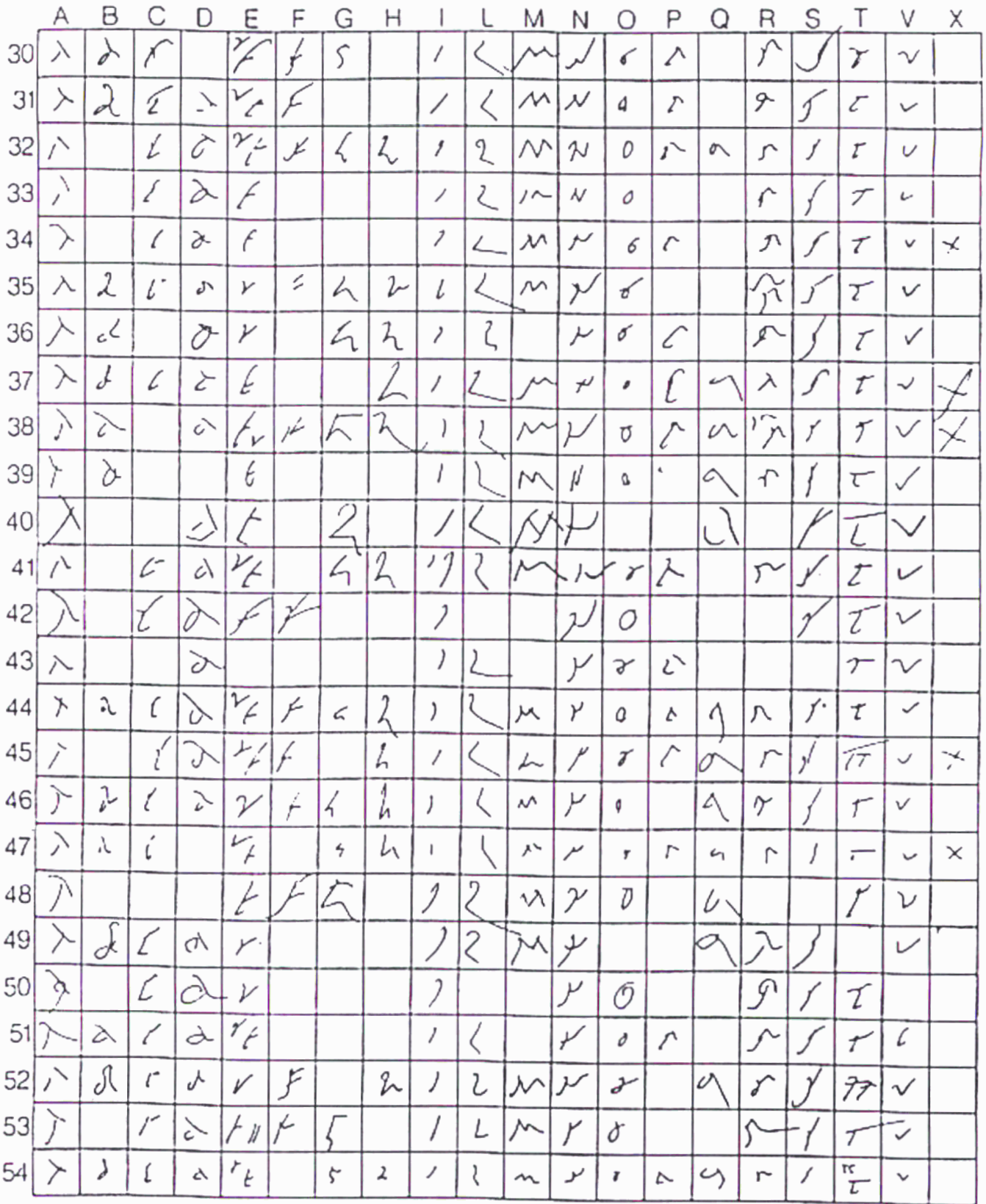

Fig. 4 - Tabulação de formas de letras (Tomlim, 1988, p. 92). 
Classica, São Paulo, v. 11/12, n. 11/12, p. 213-230, 1998/1999.

221

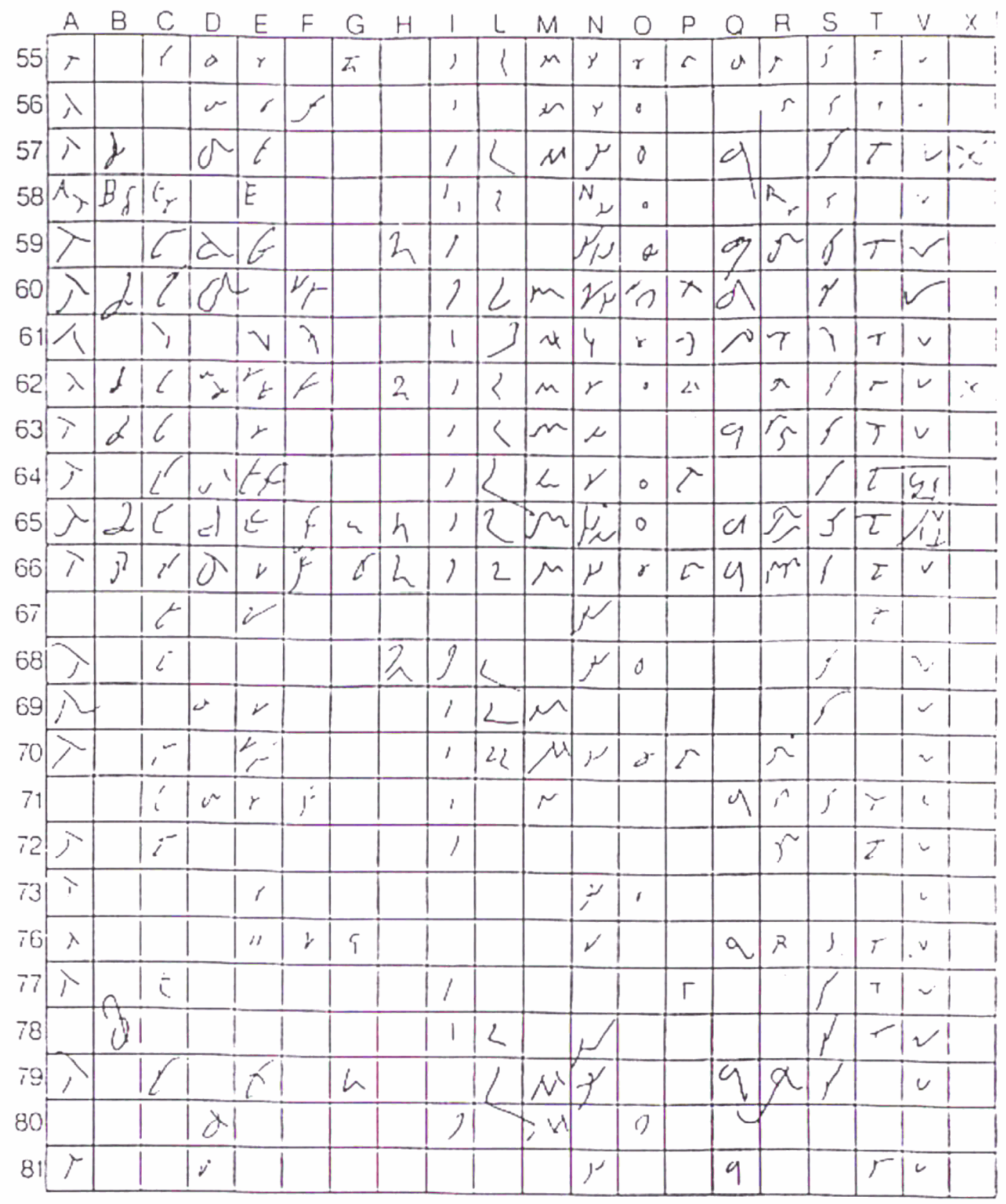

Fig. 5 - Tabulação de formas de letras (Tomlim, 1988, p. 93). 


\begin{tabular}{|c|c|c|c|c|c|c|c|c|c|c|c|c|c|c|c|c|c|c|c|}
\hline A & $B$ & C & D & $E$ & $F$ & $G$ & $\mathrm{H}$ & & $L$ & $M$ & $\mathrm{~N}$ & 0 & $P$ & $Q$ & $R$ & $S$ & $T$ & V & \\
\hline 5 & & $c$ & 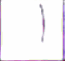 & $1^{\prime}$ & & & & 1 & & $m$ & & 0 & & & & $v$ & & & \\
\hline j & & $r$ & & $F$ & & & & 1 & 1 & $\alpha$ & $\gamma$ & 0 & $\lambda$ & $-V$ & & 12 & 5 & $\cup$ & \\
\hline & 2 & $c$ & $d$ & $c_{1}$ & $f$ & & $h$ & 1 & 3 & $m$ & $\mu$ & 0 & & $a$ & $J$ & $J i$ & 2 & $u^{\prime}$ & \\
\hline & & & & $q^{2}$ & & & & & & & & & $P$ & & $r y$ & 7 & & $u$ & \\
\hline$\lambda$ & & $r$ & 9 & 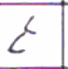 & $l^{6}$ & 5 & & 1 & 3 & $m$ & $H_{\lambda}$ & 0 & 1 & 9 & \begin{tabular}{l|l}
$n$ \\
\end{tabular} & $Y$ & $T$ & 4 & $x$ \\
\hline$\not x$ & $b$ & $c$ & $d$ & $\epsilon_{6}$ & & & & 1 & 2 & $m$ & $N$ & 0 & & & $R$ & $r$ & & 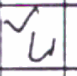 & \\
\hline$\pi$ & 4 & $C$ & $d$ & $\epsilon$ & & & & 1 & $L$ & $m$ & $N$ & 0 & $p$ & & $R$ & 5 & $\tau$ & U & \\
\hline a & $b$ & r & $d$ & $t$ & K & $S$ & h & 1 & 2 & $m$ & $\begin{array}{c} \\
n \\
\end{array}$ & a & $\Gamma$ & 9 & $n r$ & $r_{1}$ & $\tau$ & 4 & $x$ \\
\hline$n$ & $b \&$ & $\zeta$ & $d$ & $E$ & $F$ & $\zeta$ & $h$ & 1 & $l_{L}$ & $m$ & $n$ & 0 & $p$ & 7 & \begin{tabular}{|l|}
$r$ \\
\end{tabular} & $\gamma$ & $\tau$ & n & $x$ \\
\hline & & $C$ & d) & $\varepsilon$ & & $S$ & $h$ & 1 & $L$ & $m$ & $N$ & 0 & $P$ & 9 & $r_{r}$ & $r$ & $\tau$ & 4 & $x$ \\
\hline & L & 7 & i & ᄂ & f & 5 & $n$ & 1 & $L$ & $m$ & $\begin{array}{l}u \\
n\end{array}$ & $a$ & 12 & 4 & $\psi$ & $Y$ & $T$ & $u$ & \\
\hline & & & & 5 & & 5 & & 1 & $\lambda$ & $m$ & $n$ & & $P$ & & $r$ & $\gamma$ & $\tau$ & 4 & \\
\hline & & & & E & $f$ & & & 1 & $<$ & $m$ & $H$ & & 1) & & $r$ & $v$ & $\tau$ & 4 & \\
\hline & & $c$ & a & $\angle E$ & $F<$ & & & 1 & & $n$ & $D$ & 0 & $p$ & & $Y^{\prime}$ & $Y j$ & $n_{1} T$ & 4 & \\
\hline$\lambda$ & & $\zeta$ & & & $F$ & & & 1 & & $m$ & $n$ & () & & & & $\gamma$ & & $U$ & \\
\hline & & $i^{\prime}$ & $J$ & & $v^{2}$ & 5 & & 1 & $<$ & $m$ & $n$ & $\gamma$ & $p$ & & \begin{tabular}{|l|l|}
$r$ & $Y$ \\
\end{tabular} & $\gamma$ & $t$ & $\sim$ & \\
\hline & & & & & & & & 1 & & $M$ & & & & $a$ & & & & & $(1$ \\
\hline
\end{tabular}

Fig. 6 - A epigrafia das ânforas oleárias béticas. (Tomlin, 1988, p. 94). 


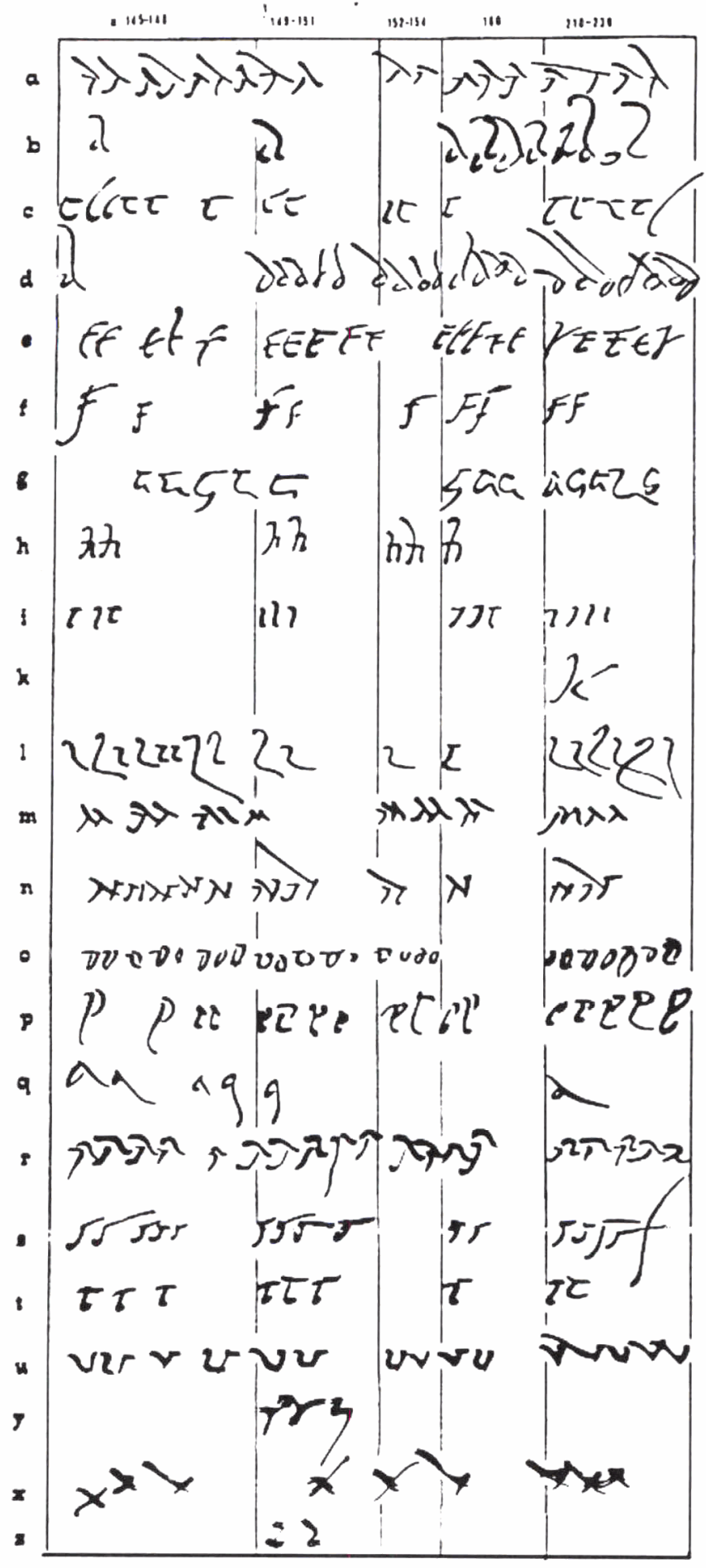

Forme più comuni delle lettere dellálfateto corsivo adoperato nei controlli delle anfore Dr. 20

Fig. 7 - Rodríguez-Almeida 1984, fig 93. 
224

Pedro Paulo A. Funari: A escrita cursiva romana.

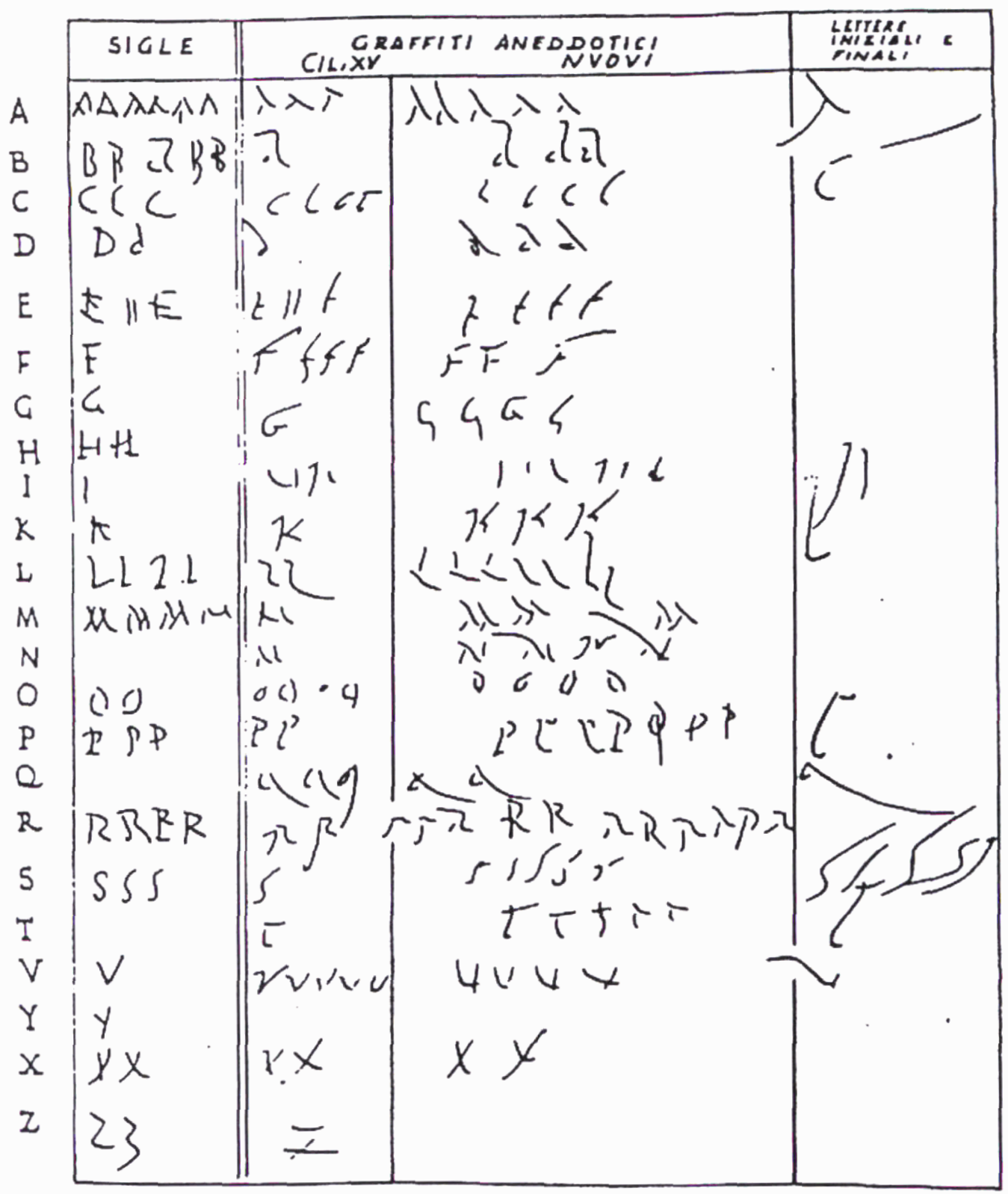

Fig. 8 - Rodríguez-Almeida 1984, fig 83. 
Classica, São Paulo, v. 11/12, n. 11/12, p. 213-230, 1998/1999.

225
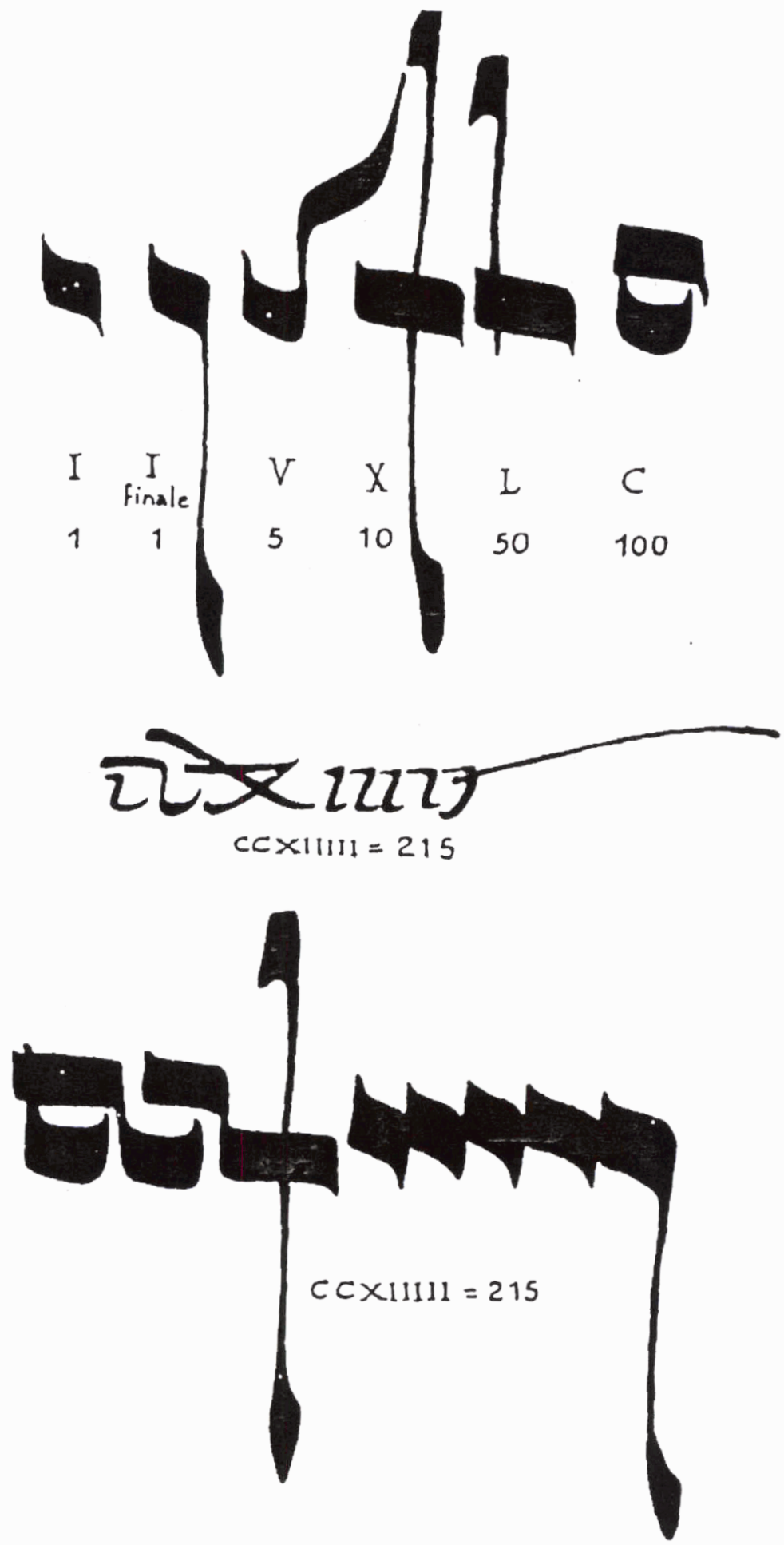

Fig. 9 - Rodríguez-Almeida 1984, fig 74. 

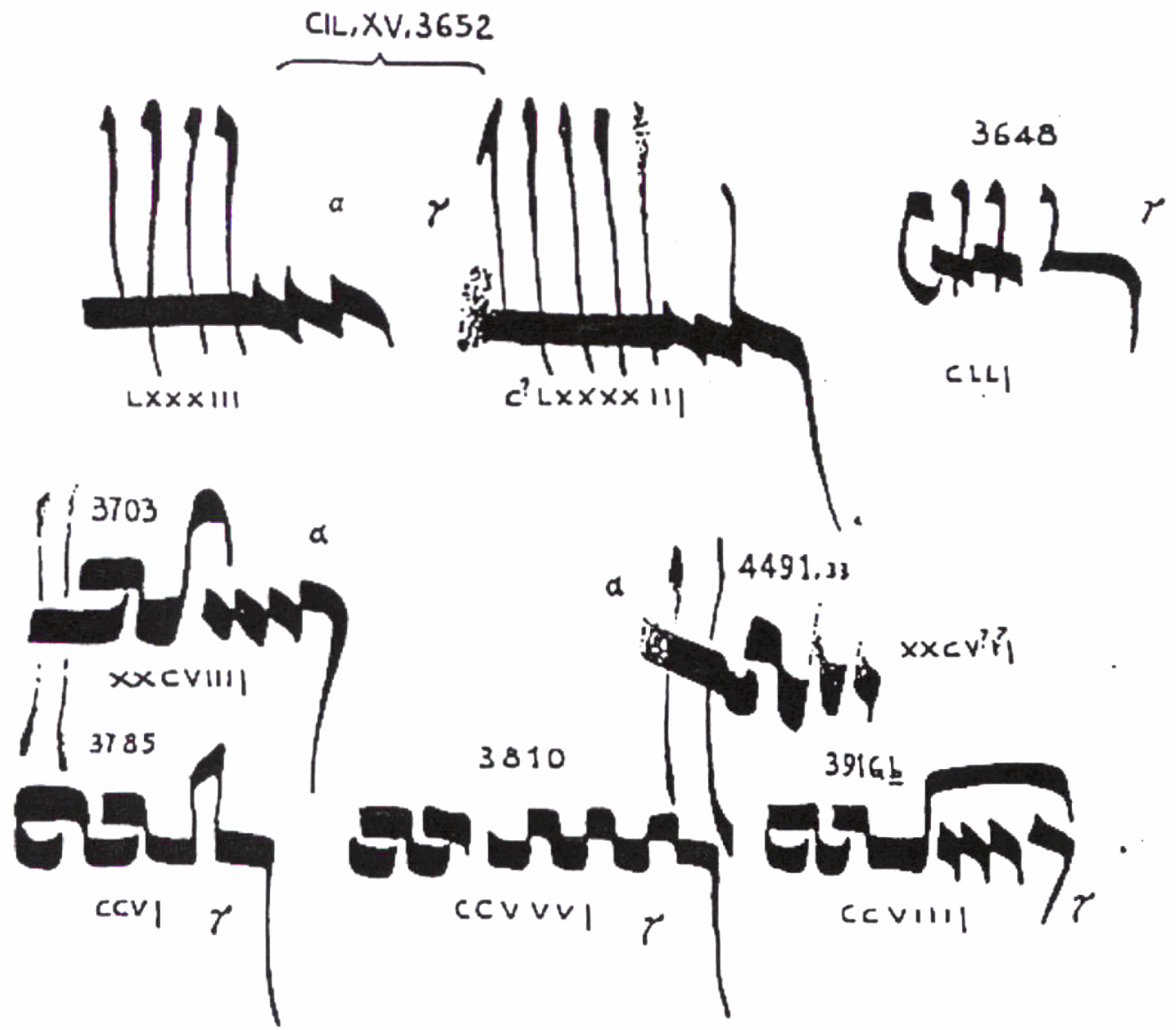

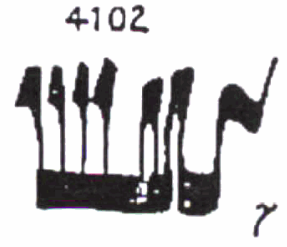

LLLLVVV

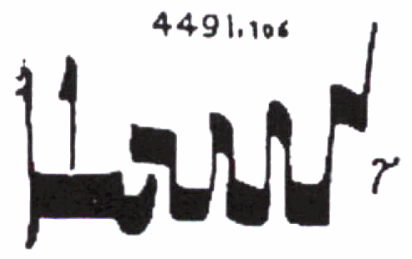

LLCVYV

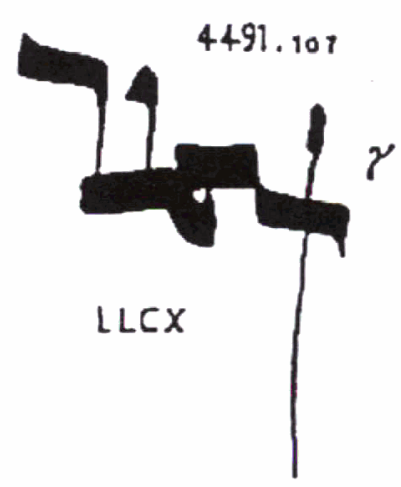

Fig. 10 - Rodríguez-Almeida 1984, fig 75. 

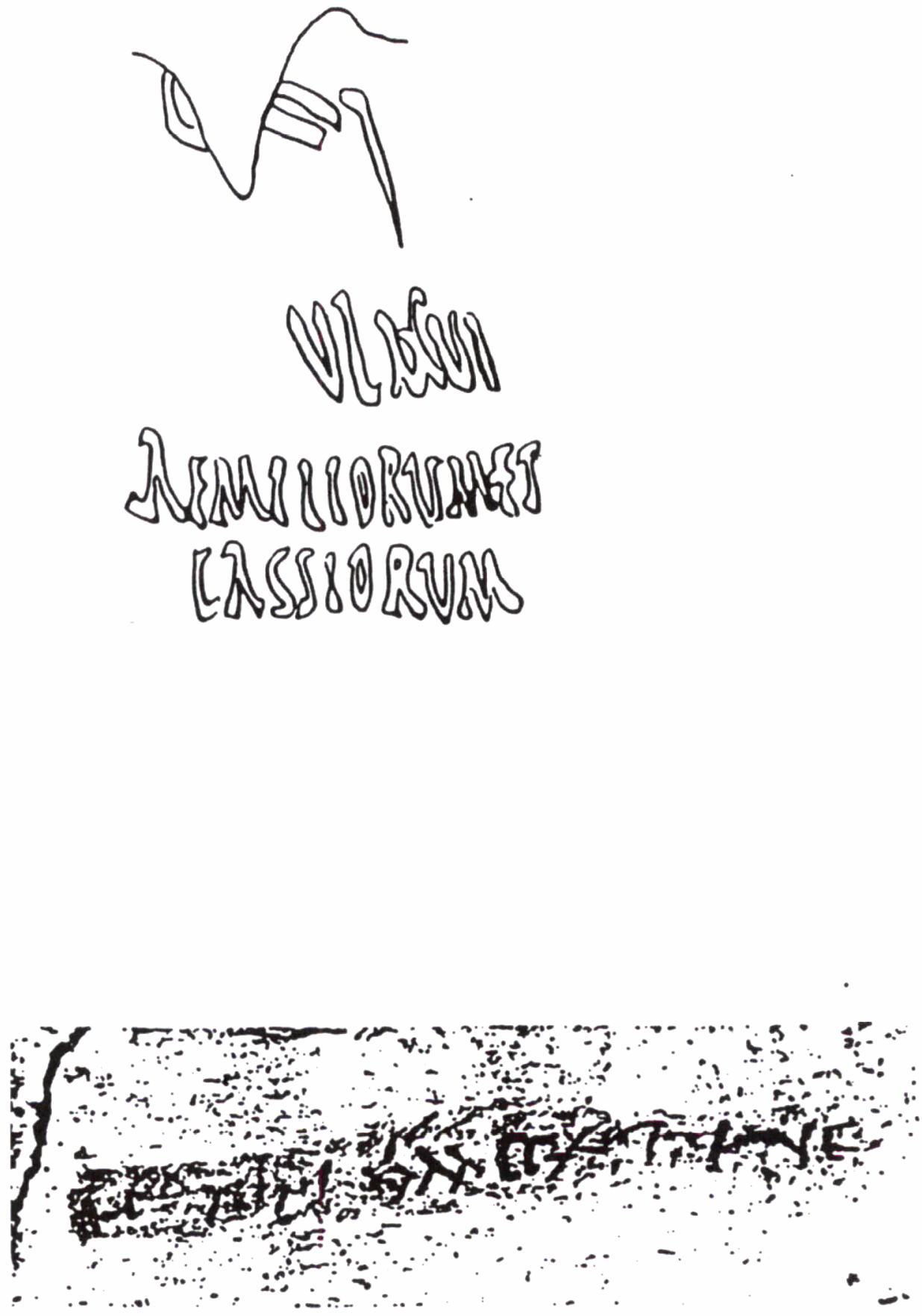

Fig. 12 - Funari 1991, fig 12.6. 


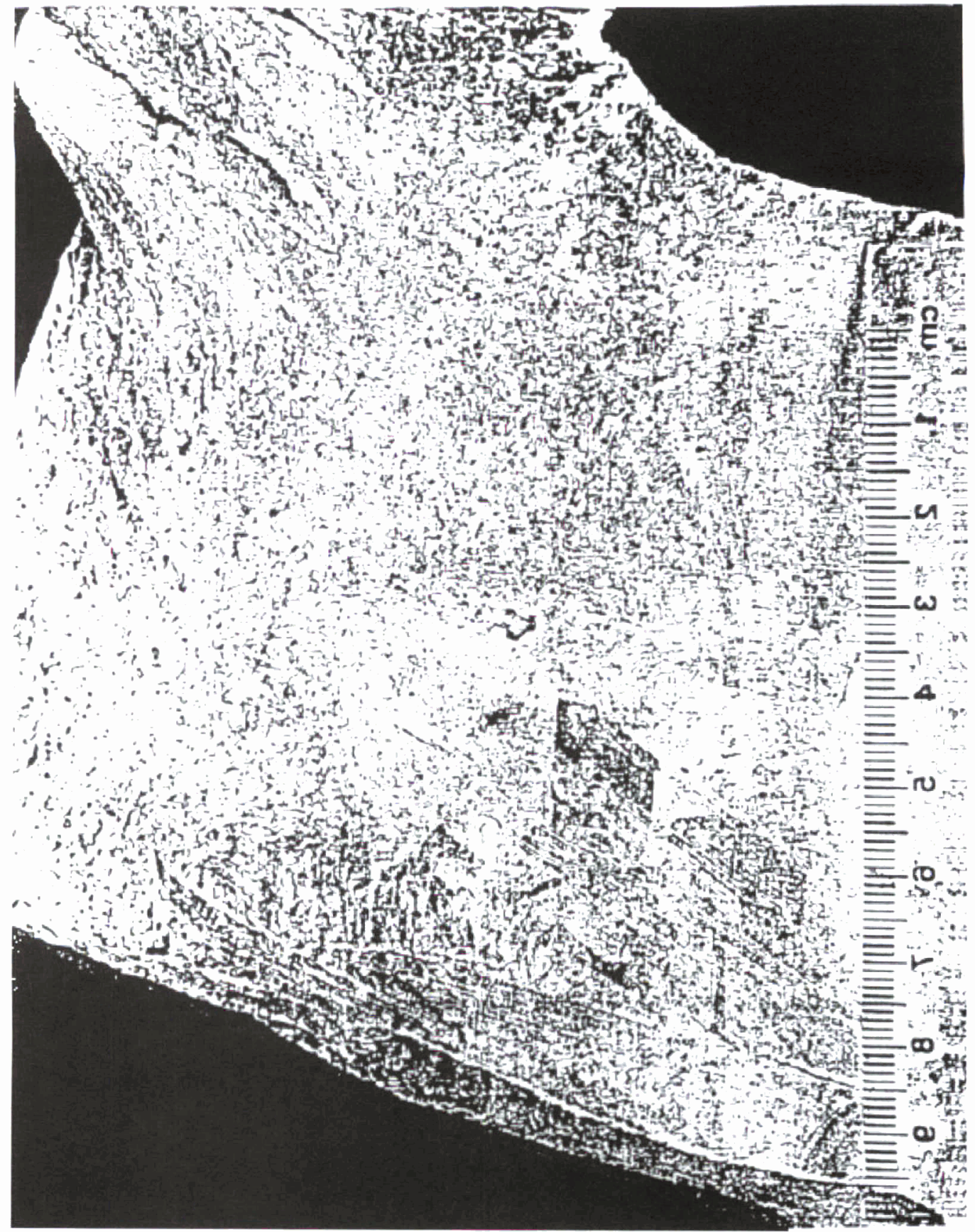

Fig. 13 - Foto inédita da inscrição proveniente de Catterick, Inglaterra, estudada por Funari (Funari, 1993, fig. 9) 


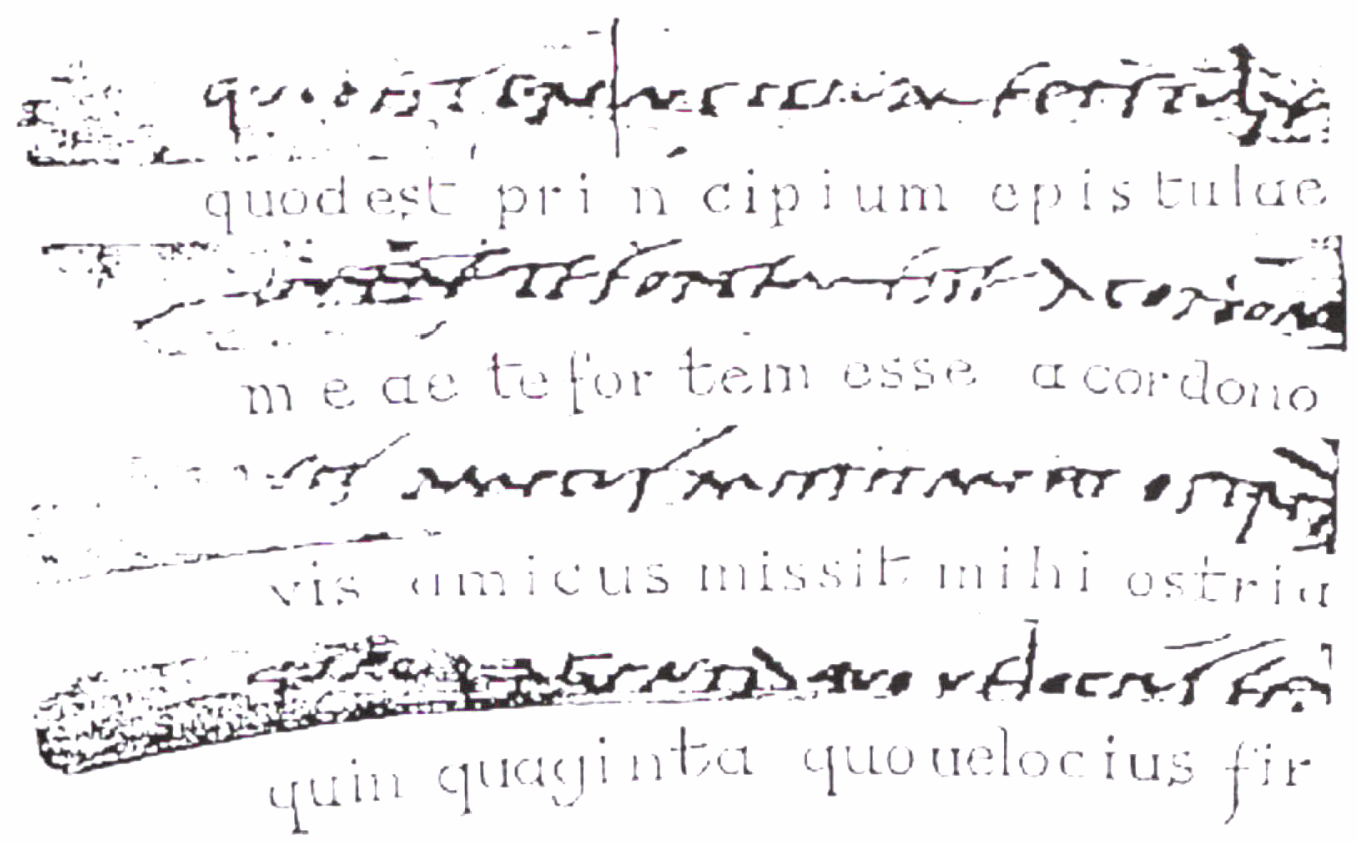

25 Table 6. Scale i: 2: 3

quod est principium epistulae meae te fortem esse. a Cordonouis amicus missit mihi ostria quinquaginta. quo uelocius fir

Back Lucio decurion[i]
... which is the first point of my letter (to hope?) that you are vigorous. From Cordonovi a friend has sent me fifty oysters. In order that ... more speedily...

Back To Lucius, decurion

Fig. 14 - Carta proveniente de Vindolanda (Bowman, 1983, fig. 25). 


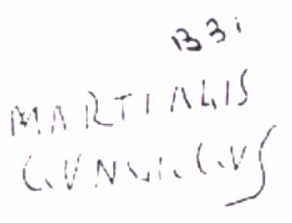

ab occidente, longa 0,105 .

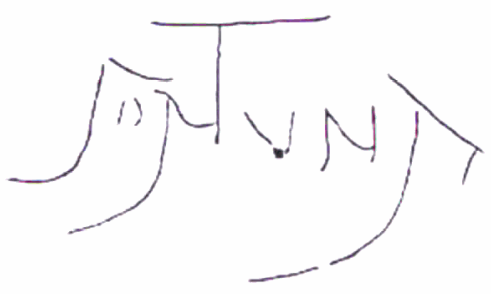

scripsi et edidi Bull. Inst. 1882 n. 218 n. 8. - Fortuna
4764 infra 4763, longa 0,035 .

\section{Descripsimus; dedi ex apographo meo charta perlucida exarato. Petra Giorn. d. sc. II p. 226 n.43; Zangemeister Epb I. n. 2801 PERAM De Petra; PERARL Zangemeister; sane ultima litera \\ L esse videtur; quin tamen I voluerit scriptor, vix dubitari potest Perati fur es.}

$\mathbf{4 7 5 5}$ ad d. 4454, scrpta in similitudinen navis, longa 0,07.

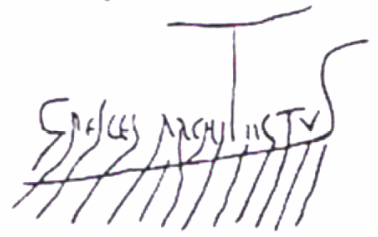

Descripsimus; dedi ex apographo meo charta perlucida exarato. D. Petra Giorn.d. sc. II p. 225 n. 17; Zangemeister Eph I. n. 274. Cresces architectus.

$\mathbf{5 3 7 2}$ prope angulum sinistrum, in tectorio rubro, longa 0,12 .

4756 in tectorio rubro, inter occidentem et meridiem; longa 0,09.

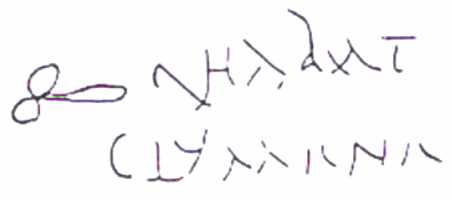

Descripsimus; dedi ex apographo meo charta perlucida exarato. HABIT EX HABIIT correctum. HABIIT CLYMII NA, om. a De Petra Giorn. d. sc. II p. 225 n.18; q HABIAT CLYMiINII Zangemeister Eph n. 275, qui interpretatur: Q(uintus) hab(e)at Clymene(n). Intellegas potius habeat Clymene scil. phallum.

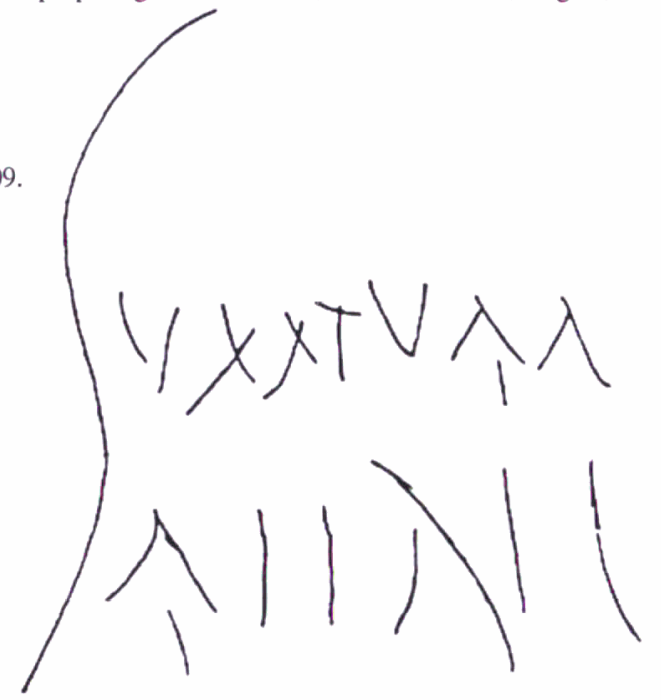

Dedi ex apographo meo charta perlucida exarato. - Edidi Bull. 1883 p. 75 n. 1; Sogrliano Not. d. sc. 1880 p. 488.

1 A fortasse alienum; om. Sogliano. -2 om. Sogl.; AlIRII Mith Sum tua ae(ris) a(ssibus) II?

Fig. 15 - Apógrafos do Corpus Inscriptionun Latinarum IV. 